\title{
Initial time-resolved particle beam profile measurements at the Advanced Photon Source
}

\author{
B. X. Yang and A. H. Lumpkin

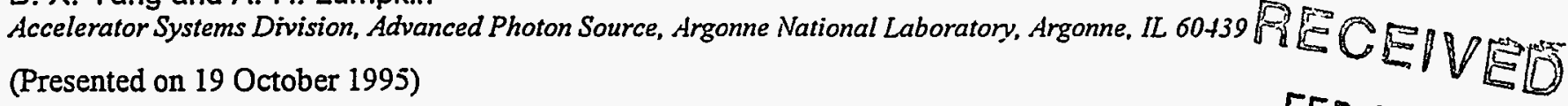 \\ The commissioning of the 7-GeV Advanced Photon Source (APS) storage ring began in early \\ 1995. Characterization of the stored particle beam properties involved time-resolved transverse and \\ longitudinal profile measurements using optical synchrotron radiation (OSR) monitors. Early \\ FFe $\cap 81996$
} results include the observation of the beam on a single turn, measurements of the transverse beam sizes after damping using a $100 \mu \mathrm{s}$ integration time $\left(\sigma_{x} \sim 150 \pm 25 \mu \mathrm{m}, \sigma_{y} \sim 65 \pm 25 \mu \mathrm{m}\right.$, depending on vertical coupling), and measurement of the bunch length ( $\sigma_{\tau} \sim 25$ to $55 \mathrm{ps}$, depending on the charge per bunch). The results are consistent with specifications and predictions based on the $8.2 \mathrm{~nm}$-rad natural emittance, the calculated lattice parameters, and vertical coupling less than $10 \%$. The novel, single-element focusing mirror for the photon transport line and the dual-sweep streak camera techniques which allow tum-by-tum measurements will also be presented. The latter measurements are believed to be the first of their kind on a storage ring in the USA. (C) 1995 American Institute of Physics

\section{INTRODUCTION}

The 7-GeV storage ring at the Advanced Photon Source (APS) began commissioning in early 1995 . Measurements of the transverse sizes and the bunch length of the stored beam contribute to characterizing transverse and longitudinal emittance, respectively. For this purpose, imaging with optical synchrotron radiation has been used in the storage rings of most synchrotron radiation sources. There are fundamental limitations for obtaining high spatial resolution with this approach:

(1) The diffraction limit. Due to relativistic motion of orbiting electrons or positrons, the synchrotron radiation concentrates strongly in the forward direction, with an rms angular radius defined by.

$$
\sigma_{p y 0}^{\prime}=\frac{1.07}{\gamma}\left(\frac{\lambda}{\lambda_{c}}\right)^{1 / 3}=1.22\left(\frac{\lambda}{f}\right)^{1 / 3},(p=39.8 m) .
$$

Hence the diffraction limit for spatial resolution is

$$
\sigma_{p y}^{o s r}[\mu u n] \approx \frac{\hbar[\mu m] / 2}{\sigma_{p y 0}^{\prime}[\mathrm{rad}]}=+1 \lambda^{2 / 3}[\mu m] .
$$

(2) The curred positron orbit and the finite source depth acceptance contribute to the transterse image size.

The minimization of the combined image size due to the above effects' leads to the optimal acceptance angle

$$
\sigma_{i, 2}=\left(\frac{\lambda}{p}\right)^{1,3} \approx 1 \mathrm{mrad} \text {. }
$$

and the optimum resolution

$$
\sigma_{y R} \approx\left(\rho \lambda^{2}\right)^{1,3} \text {, or } \sigma_{s R}[\mu m] \approx 100 \lambda_{i}=3[\mu m] \text {. }
$$

For the shortest wavelength we use $\lambda_{2}=0.25 \mu \mathrm{m}$ and $\sigma_{y R}=10 \mu \mathrm{m}$. It was expected that this resolution would be

\begin{tabular}{|c|c|}
\hline Beam natural emittance & $8.2 \mathrm{~nm} \cdot \mathrm{rad}$ \\
\hline Vertical coupling & $10 \%$ \\
\hline Energy spread & $-0.1 \%$ \\
\hline Dispersion & $92 \mu \mathrm{m} / 0.1 \% \Delta \mathrm{E} / \mathrm{E}(\mathrm{H})$ \\
\hline rms betatron oscillation & $114 \mu \mathrm{m}(\mathrm{H}) \times 117 \mu \mathrm{m}(\mathrm{V})$ \\
\hline Total beam size & $146 \mu \mathrm{m}(\mathrm{H}) \times 117 \mu \mathrm{m}(\mathrm{V})$ \\
\hline
\end{tabular}
sufficient for the designed operation conditions (see Table 1).
Table 1 Design Parameters for the APS Bending Magnet Sources ${ }^{2}$

In this report, we discuss the design and characterization of the APS UV/visible diagnostics beamline and present initial results obtained from its operation.

\section{BEAMLINE DESIGN}

To obtain the optimum resolution, we face a serious challenge in the optics design: operating diffraction-limited optics under high heat load.

The distance from the bending magnet source to the first optical element could not be shorter than $13 \mathrm{~m}$ due to space limitations in the storage ring tunnel. Hence the rms angular resolution required of the primary optical system is $3 \mu \mathrm{rad}$ or better (measured from the first optical element). In this resolution regime we face all the technical challenges astronomers face in designing high precision telescopes: refractive optical elements (lenses) are not suitable since the chromatic aberration alone exceeds the tolerance: all optical surfaces have to be stringently figured and polished: and fluctuations due to air currents must be reduced by evacuated transport pipes. The estimate of the impact of mirror surface distortion on the image yields a total tolerance budget for the entire mirror imaging șstem:

$$
\text { RMIS DISTORTION } \leq \frac{\sigma_{1 \kappa} \cdot \sigma^{\prime}:}{2} \approx \frac{\lambda}{2} \approx 20 \mathrm{~mm} .
$$

(C) 1995 American Institute of Physics 1 

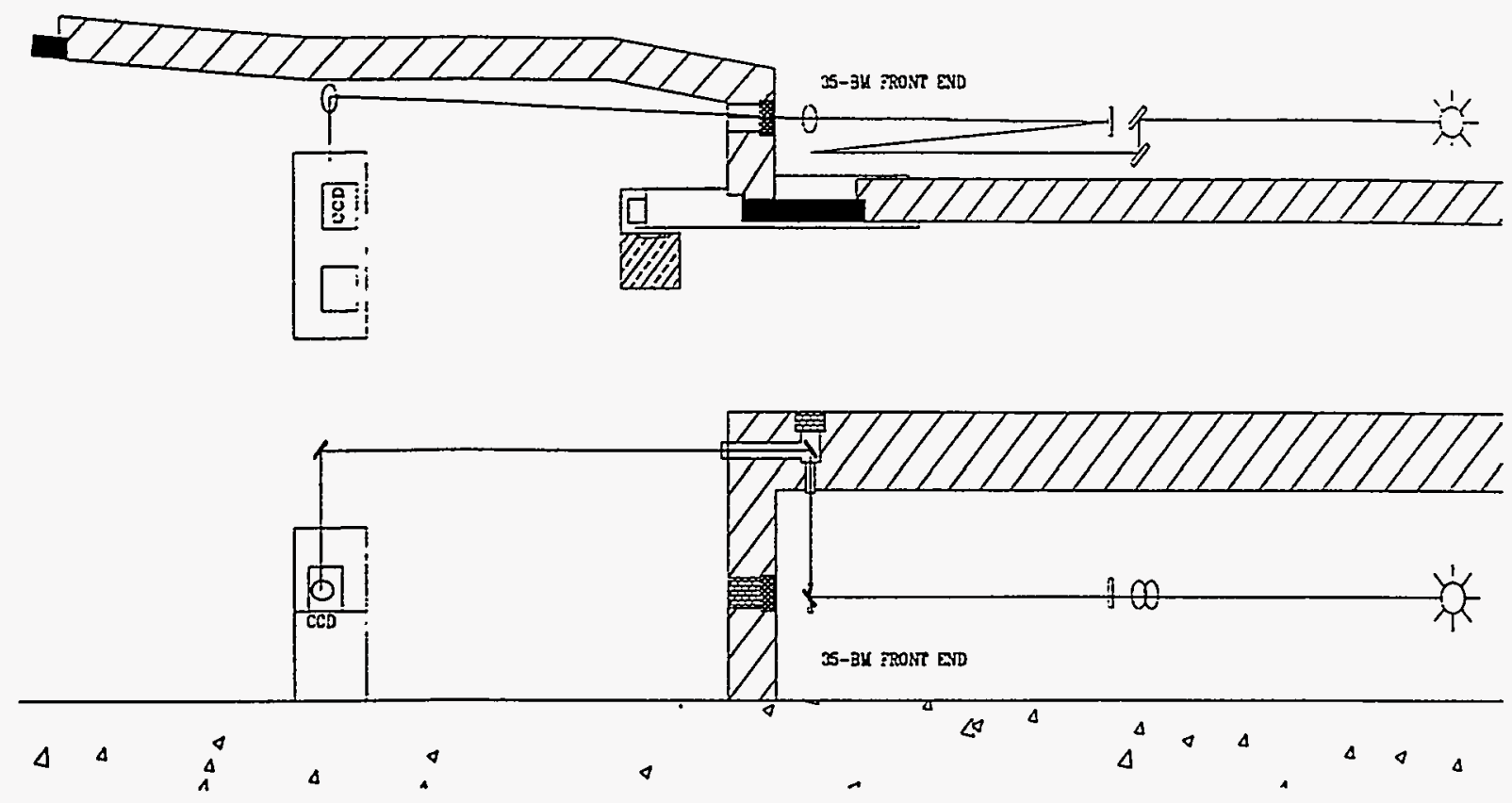

Fig. 1 Schematics of the UV/visible beam transport of the diagnostics beamline of the Advanced Photon Source. A number of planar mirrors are used to lead the beam out of the radiation environment to the optics lab.

This is equivalent to a peak-to-peak figure error of $\lambda / 13$ at $632 \mathrm{~nm}$, which is obtainable with the high end optical elements.

The power load under the nominal operating condition $(7 \mathrm{GeV} / 300 \mathrm{~mA})$ is $260 \mathrm{~W}$ per horizontal milliradian or $20 \mathrm{~W} / \mathrm{mm}$, under which no optical elements can maintain the stringent requirement of Eq. (5). We took advantage of the fact that the UV/visible radiation spreads much wider vertically than the $x$-ray radiation which carries the bulk of the power, and designed a slotted mirror ${ }^{3}$ that can pick off the UV/visible light for the beamline without being heated up excessively. Initial data presented here, however, were taken with an uncoated molybdenum mirror (Melles Griot) mounted on a water-cooled backplate before the slotted mirror was available. This interim mirror has been used with up to $10 \mathrm{~mA}$ beam current without seeing significant distortions.

Functionally, the beamline is divided in two parts: the primary and secondary optics. The primary optics relay the image into an optics lab at about 8:7 magnification. The image is then read out in parallel by a charge-coupled device (CCD) camera directly and by sereral other detectors and cameras through beam splitters and lenses. Since the image from the primary system will be about $1-2 \mathrm{~m}$ from these cameras, the angular resolution requirement for these camera optics is relaxed to about $20 \mu \mathrm{rad}$. High quality commercial optics, in conjunction with bandpass filters. are used to meet the requirement.

The major imaging element of the primary optics is a concave spherical mirror. Optical ray tracing was used to evaluate the rms radius of the image spot (point spread function). For the apertures consistent with Eq. (3), the spot size follows well with an analytical expression, $R[\mu \mathrm{m}]=20 \theta^{2}[\mathrm{DEG}]$, where $\theta$ is the incidence angle. An incidence angle of 1 degree was chosen for the spherical mirror from this analysis. Figure 1 shows the schematics of the primary optics.

\section{TRANSVERSE BEAM SIZE MEASUREMENTS}

The transverse profile of the storage ring beam was measured by placing a high resolution CCD camera (Vicon $2410,1 / 2$ " format with $768 \times 494$ square pixels) at the focal point of the primary optics. The exposure time of the camera is normally set electronically to $100 \mu \mathrm{s}$, equivalent to the time an electron makes 27 turns in the ring. The transverse length scale $(7.6 \mu \mathrm{m}$ per pixel) was calibrated with a retractable. illuminated microscopy resolution target (Geller Microanalytical Laboratory, Model MIRS-3) placed at the source location. Analyzing the intensity variations of the image lines of the target allowed us to estimate the total geometrical aberration and hence the resolution of the optical system. We concluded that the radius of the point spread function due to aberration is less than $26 \mu \mathrm{m}$. which is to be added in quadrnture to that given by. Eq. (2).

In the early stage of the storage ring commissioning. it was possible to capture the image of the electron beam eren when it passed the source point only once. Figure 2 shows such an image taken when the beam was injected to the ring but blocked by a flag before completing a turn (.) Warch IS. 1995). Figure 3 shows the first stored beam in the APS at $4.5 \mathrm{GeV}$. where the vertical size is much larger than the horizontal one due to the lattice parameters used (March 25. 1995). 


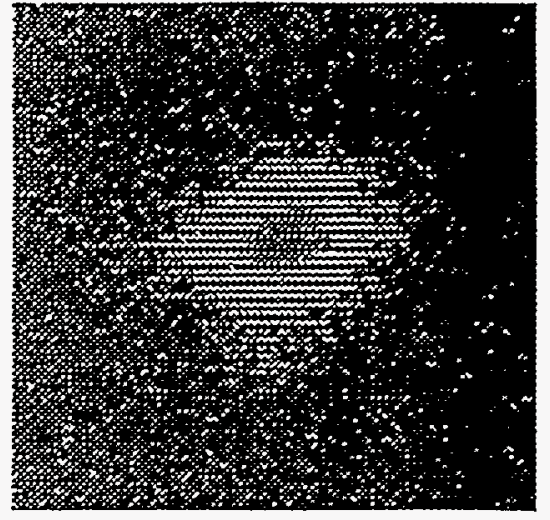

Fig. 2 The electron bcur: sn . Jurch 18. 1995. Tris picture was taken with low resolution d!!c !o the large beam size (undamped).

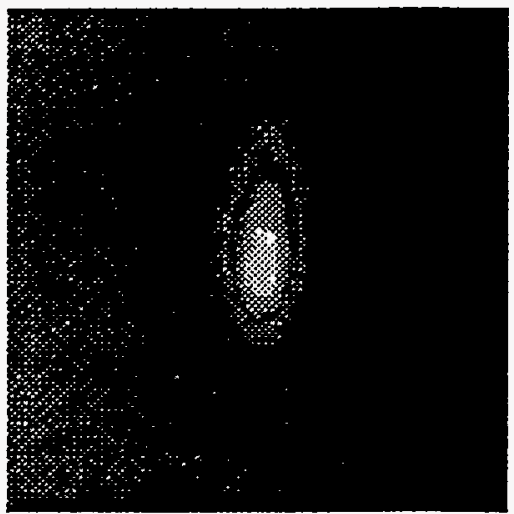

Fig. 3 The image of the tirst stored electron beam at $4.5 \mathrm{GeV}$ in

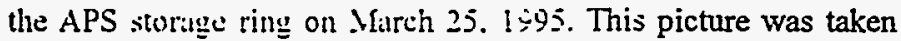
with lower resolution due to the large seam size.

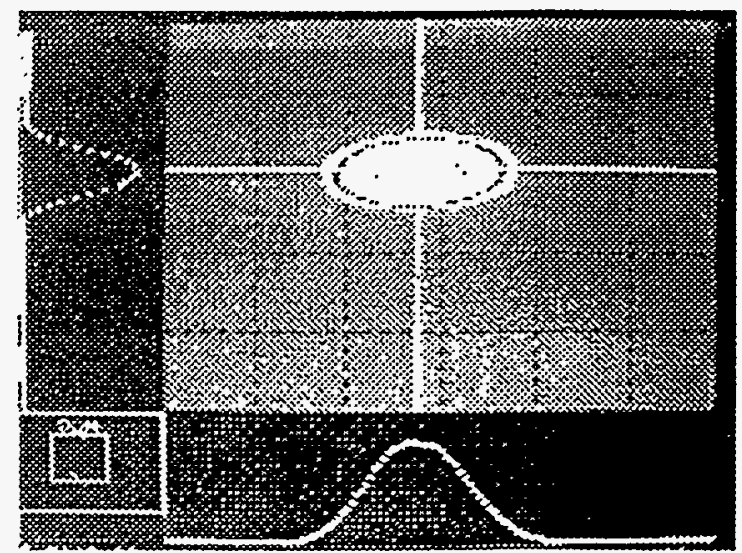

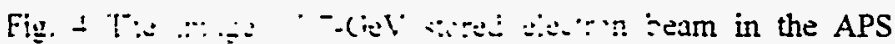

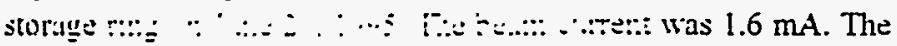

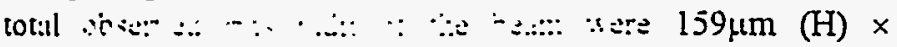
$68+111 \%$

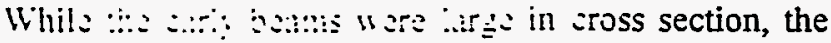

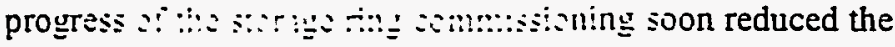

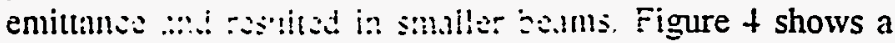

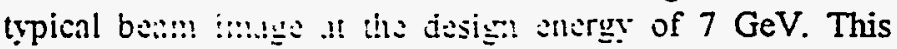

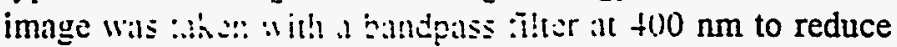

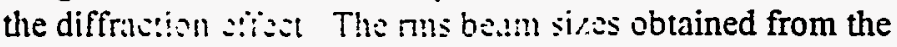

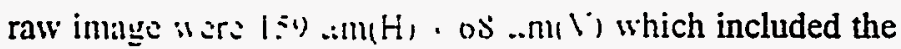
Rev. Sci. Instrum. 66 (9). September 1995 resolution contribution of about $60 \mu \mathrm{m}$. After subtracting the resolution contribution. we obtained beam sizes of $147 \mu \mathrm{m}(\mathrm{H}) \times 32 \mu \mathrm{m}(\mathrm{V})$. which were within the design values given in Table $I$ and consistent with the emittance of the storage ring meeting its design goal at low current. The vertical beam size was much less than the design value because a lower vertical coupling was used. Since the size was about half of the instrument resolution. a large error bar was assigned to it. It was apparent that as we reached the diffraction limit of the UV/visible imaging technique, appropriate, planned $x$-ray diagnostics techniques needed to be implemented for better characterization of the lower emittance of the ring. 5,6

\section{BUNCH-LENGTH MEASUREMENT}

The characterization of the beam bunch length is not only important to the operation of the storage ring, but also to any planned time-critical user experiments. Our measurement of the bunch length was performed with a dual-sweep streak camera (Hamamatsu C5680). The vertical sweep (Synchroscan at $117.3 \mathrm{MHz}$ ) was synchronized with the storage ring if source $(352 \mathrm{MHz})$. The horizontal scan was triggered from a local oscillator but may be synchronized with the injector during studies of the injection and damping process. By sweeping in both directions, it was possible to perform bunch-length measurements on a turnby-turn basis, and to differentiate individual bunches circulating in the ring, if they were sufficiently far apart.

We noticed significant phase motion of the stored beam, at various frequencies, which broadens the bunch length significantly if signals from different turns are integrated (Synchroscan alone). Much of this phase motion was linked to the fluctuations of the if power supply of the ring. Hence only data from turn-by-turn measurements will be presented here.

Figure 5 shows the sideview of a single bunch from a typical dual sweep. In setting up this experiment, the beam image was rotated 90 degrees. Hence the horizontal width of the image actually shows the vertical size of the beam (see the top scale of the figure), while the vertical dimension of each bunch image shows the beam length in time $\left(\sigma_{\tau}-45 \mathrm{ps}\right)$. To read the time between passes. the lower horizontal scale should be used.

Figure 6 shows the dual sweep image of a beam in a head-to-tail instability ( $7 \mathrm{nC} / \mathrm{bunch}$ ) purposely created by reducing the strengh of the sextupole magnets. It can be seen that the bunches are not only enlarged rertically. but also skewed. i.e.. the head and tail of the bunch were abore and below the mid-orbit plane. respectively. The bunch images repeat approximately every three turns. corresponding well to the fraction of the vertical tune $(1+.3 /$ turn). 


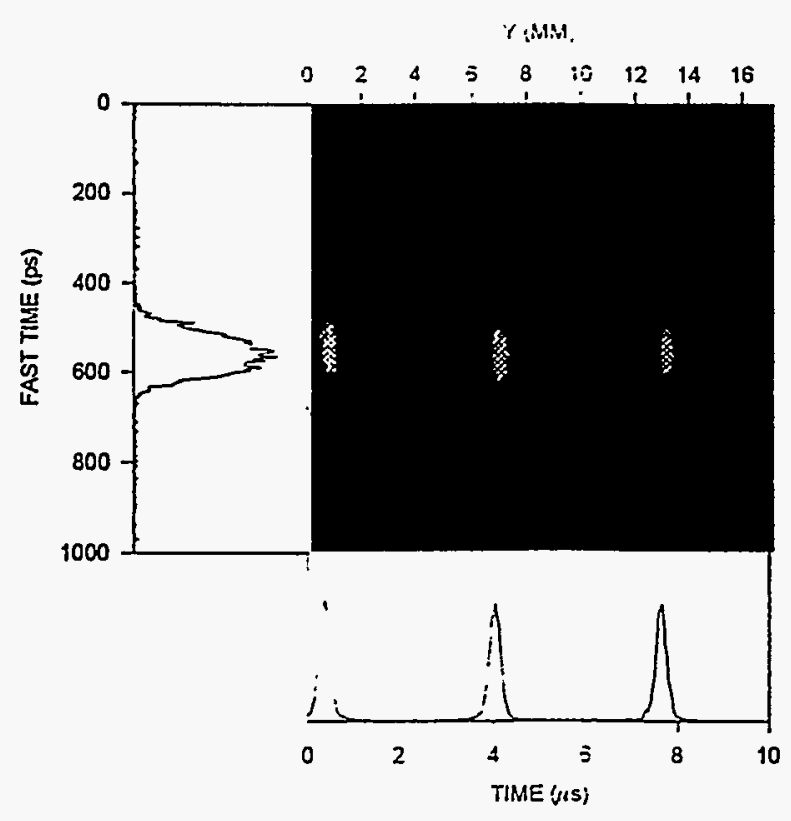

Fig. 5 A typical dual sweep image

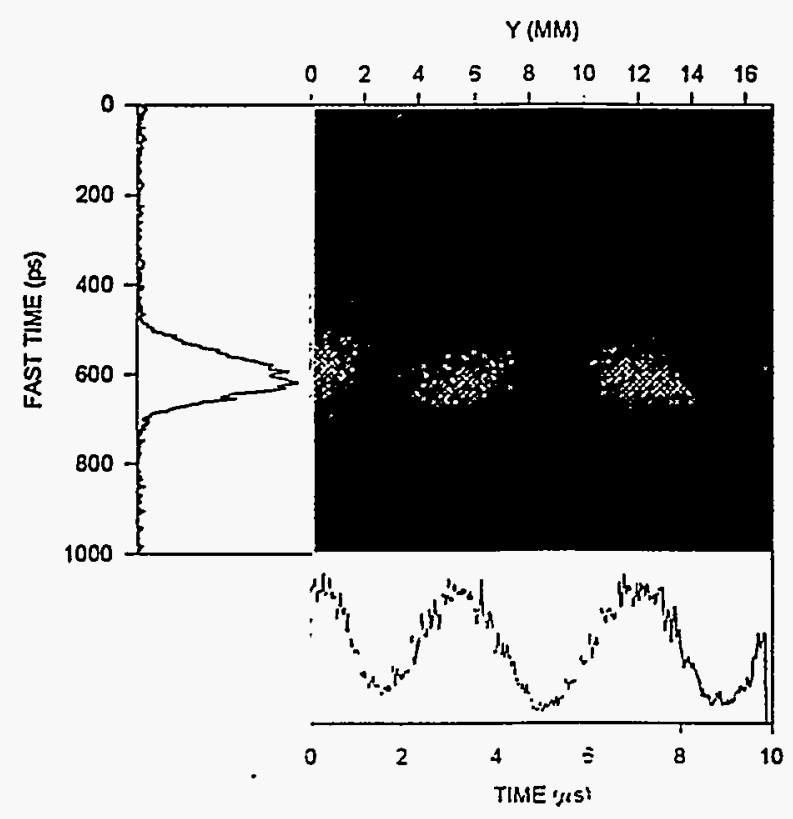

Fig. 6 Dual sweep image of a beam with a vertical head-to-tail instability.

\section{CONCLUSION}

The UV/risible diagnostizs beamiine for the APS storage ring has been constructed and tested at low beam current (up to $10 \mathrm{~m} . \lambda$ ). It has achieved nearly ideal resolution at $400 \mathrm{~nm}$ wavelength. The ker to its success is a novel spherical mirror imagingirelay ș̣stem.

The transverse beam profile measurements show that the beam sizes are consistent with the APS storage ring's emittance objectives. but under low cutrent conditions and with vertical coupling less than the design goal of $10 \%$.

The capability of measuring beam length simultaneously with one of its transterse dimensions with good temporal and spatial resolution has been demonstrated. This has great potential in studies of fast beam dynamics of low emittance beams of the third-generation synchrotron radiation sources, as the data on the head-to-tail instability have shown.

Further characterization and tests are in progress as the storage ring beam current approaches its design goal of $100 \mathrm{~mA}$.

\section{ACKNOWLEDGMENTS}

We would like to thank G. E. Brown for many stimulating discussions; E. Rotela, I. C. Sheng, S. Sharma, and D. Walters for their support in the mechanical and vacuum design of the beamline; $M$. Borland, G. Decker, L. Emery, and S. Milton for their help in accelerator physics. This work was supported by the U.S. Department of Energy, Office of Basic Energy Sciences, under Contract No. W-31-109-ENG-38.

${ }^{\prime}$ R. C. Nawrocky, J. Galayda, L. H. Yu, D. M. Shu, IEEE Trans. on Nucl. Sci., NS, No. 5, 1893 (1985).

${ }^{2}$ Glenn Decker, private communications.

${ }^{3}$ E. R. Rotela, B. X. Yäng, I. C. Sheng, S. K. Sharma, and A. H. Lumpkin, "High precision, high heat-load mirror for the APS diagnostics beamline," these proceedings.

${ }^{4}$ A. Lumpkin and B. Yang. "Status of the Synchrotron Radiation Monitor for the APS Facility Rings," Proceedings of the 1995 Particle Accelerator Conference, Dallas, Tx, May 1-5, 1995, to be published.

${ }^{5}$ P. Elleaume, C. Fortgang, C. Penel, and E. Tarazona, "Measuring ultra small electron emittance using an X-ray pinhole camera," ESRF/MACH ID 95/25.

${ }^{6}$ R C. C. Perera, M. E. Melczer, A. Warwick, A. Jackson, and B. M. Kincaid. "Diagnostic beam line for a third generation storage ring," Rev. Sci. Instrum. 63, 541 (1992). 


\section{DISCLAIMER}

This report was prepared as an account of work sponsored by an agency of the United States Government. Neither the United States Government nor any agency thereof, nor any of their employees, makes any warranty, express or implied, or assumes any legal liability or responsibility for the accuracy, completeness, or usefulness of any information, apparatus, product, or process disclosed, or represents that its use would not infringe privately owned rights. Reference herein to any specific commercial product, process, or service by trade name, trademark, manufacturer, or otherwise does not necessarily constitute or imply its endorsement, recommendation, or favoring by the United States Government or any agency thereof. The views and opinions of authors expressed herein do not necessarily state or reflect those of the United States Government or any agency thereof. 